Does variability in recognition memory scale with mean memory strength or encoding variability in the UVSD model?

Rory W. Spanton ${ }^{1} \&$ Christopher J. Berry ${ }^{1}$

${ }^{1}$ School of Psychology, University of Plymouth

\title{
Author Note
}

Correspondence concerning this article should be addressed to Rory W. Spanton, School of Psychology, Faculty of Health, University of Plymouth, PL4 8AA. E-mail: rory.spanton@plymouth.ac.uk

The design and analyses in this article were preregistered at the Open Science Framework and are available alongside all experimental materials, data, and analysis code at https://osf.io/wyhb2/. 


\begin{abstract}
The unequal variance signal detection (UVSD) model of recognition memory assumes that the variance of old item memory strength $\left(\sigma_{0}\right)$ is typically greater than that of new items. It has been suggested that this old item variance effect can be explained by the encoding variability hypothesis. However, Spanton and Berry (2020) failed to find evidence for this account, suggesting that $\sigma_{o}$ may simply scale with mean memory strength $(d)$ in the UVSD model. Experiments 1 and 2 examined the effects of encoding variability and strength scaling on old item variance by creating conditions in which mean memory strength and variability in item characteristics was either low or high in $2 \times 2$ factorial designs. In Experiment 1, overall strength determined estimates of $\sigma_{0}$, with no effect of item characteristic variability. The same effect of overall strength was found in Experiment 2; there was also a significant effect of item characteristic variability, although this manipulation also had some effect on $d$ and was therefore partially confounded. Experiment 3 similarly found a simultaneous increase in old item variance and memory strength in a design using mixed item characteristic variability conditions in a single study/test block. We conclude that old item variance increases with mean memory strength in the UVSD model, with uncertainty about the effects of encoding variability, and that future explanations of the old item variance effect should bear this in mind.
\end{abstract}

Keywords: Recognition Memory, Memory Strength, Encoding Variability, Strength Scaling, Unequal Variance 
Does variability in recognition memory scale with mean memory strength or encoding variability in the UVSD model?

In a recognition memory test, participants judge whether they have previously seen items in a particular context. Inevitably, some of these items are remembered better than others. This can be represented in a signal detection model wherein items at test are associated with a 'memory strength' (henceforth 'strength') variable. The strength of 'old' items (those which have been seen in a study phase) and unstudied 'new' items are represented as separate Gaussian distributions along a unidimensional continuum. Because of exposure at study, the mean of the old item distribution is generally greater than that of the new item distribution, reflecting a difference in overall strength between the two item types. The difference between these means (d) is therefore a measure of recognition performance. Recognition memory judgements are modelled by comparing the strength value of a given item to static criteria along the strength continuum that correspond to different levels of confidence that an item is either old or new. These may range from high confidence that an item is new nearer to the lower end of the continuum, to high confidence that an item is old towards the higher end of the continuum.

Although both new and old items vary in strength, it is widely accepted that the variance of the old item strength distribution $\left(\sigma_{0}\right)$ is greater than the variance of the new item distribution (see Rotello, 2017, for a review). The acceptance of this old item variance effect is motivated by analyses of the $z$-ROC, a $z$-transformed plot of the probability of correctly judging an old item "old" against the probability that a new item is incorrectly judged "old" at each level of recognition confidence in a given response scale. It is commonly found that $z$-ROCs calculated from recognition confidence data are approximately linear, with slopes less than 1 (Glanzer, Kim, Hilford, \& Adams, 1999). Since the value of the $z$-ROC slope has long been presumed to 
represent the ratio $\sigma_{\mathrm{o}} / \sigma_{\mathrm{n}}$ in a traditional Gaussian signal detection model (but see Rabe, Lindsay \& Kliegl, 2021), a non-unit $z$-ROC slope necessitates making $\sigma_{\mathrm{o}}$ a free parameter with a value typically greater than $\sigma_{\mathrm{n}}$. With this parameterization, the unequal variance signal detection (UVSD) model is defined as having parameters $\theta=\left\{d, \sigma_{0}, C_{1}, C_{2}, \ldots C_{I}\right\}$ where $I$ is the highest decision criterion level in terms of strength (Kellen, Klauer, \& Bröder, 2013). Therefore, the probability of a 'hit' response (a correct 'old' judgement) at criterion $i$ according to the model is

$$
P(H)=\Phi\left(\frac{d-C_{i}}{\sigma_{o}}\right)
$$

where $\Phi$ is the cumulative normal distribution function. The probability of a 'false alarm' response (incorrectly judging a new item 'old') at $C_{i}$ is

$$
P(F A)=\Phi\left(-C_{i}\right)
$$

Although the UVSD model can account for some commonly observed regularities in the z-ROC slope (Egan, 1958; Yonelinas \& Parks, 2007), its unequal variance assumption was created purely for the need to account for observed data, and not with a priori psychological assumptions in mind. However, a complementary psychological explanation for the unequal variance assumption was later proposed in the form of the encoding variability hypothesis (Jang, Mickes, \& Wixted, 2012; Wixted, 2007). According to this theory, the old item variance effect is caused by the presence of a large number of variables that affect memory strength at encoding. These variables contribute additional strength and variance to memory strength across a set of old items during the study phase, resulting in an increase in $\sigma_{\mathrm{o}}$ relative to $\sigma_{\mathrm{n}}$. Examples of such encoding variables could presumably include the level of attention paid to a stimulus, item characteristics, item-participant interaction, and many others. Stated mathematically, old items have some level of baseline strength, $B \sim N\left(\mu_{\text {baseline, }}, \sigma_{\text {baseline }}\right)$, which is equivalent to the new item strength distribution (Jang et al., 2012). In the study phase, $B$ is incremented by an added strength 
variable $A \sim N\left(\mu_{\text {added, }}, \sigma_{\text {added }}\right)$ during encoding. The addition of baseline and added strength gives the resulting old item distribution in the formula $O=B+A$.

There have been several attempts to test the encoding variability hypothesis and compare its predictions with those of other accounts. Koen and Yonelinas (2010) first attempted this in a method where items at study were presented for either a fixed duration of $2500 \mathrm{~ms}$, or a mixture of 1000 and $4000 \mathrm{~ms}$ durations. It was found that the latter variable encoding condition did not change estimates of $\sigma_{\mathrm{o}}$. Instead, the contribution of an additional recollection process was solely responsible for changes to the $z$-ROC slope, supposedly constituting evidence against the encoding variability hypothesis in favor of a dual-process model. However, subsequent comments by Starns, Rotello, and Ratcliff (2012) and Jang et al. (2012) clarified that these results had no bearing on the encoding variability hypothesis. This was because Koen and Yonelinas's (2010) method mixed two discrete levels of encoding strength, which would be expected to result in a mixture strength distribution rather than a Gaussian as the encoding variability hypothesis predicts. However, Koen, Aly, Wang, and Yonelinas (2013) later studied the effects of retrieval manipulations on old item variance, finding that it was possible to induce changes in estimates of $\sigma_{0}$ without manipulating encoding variability. Although this finding does not exclude the possibility that encoding variability may still have some role in determining estimates of $\sigma_{\mathrm{o}}$, it suggests that it is not the only factor that influences old item variance.

More recently, Spanton and Berry (2020) attempted to test the encoding variability hypothesis by manipulating encoding variables directly during study. To avoid the creation of mixture strength distributions that confounded Koen and Yonelinas (2010), encoding variables were manipulated by adding variance along a continuous scale, rather than by mixing two separate conditions of high or low quality encoding. Across three experiments, attempts to influence $\sigma_{\mathrm{o}}$ by manipulating three encoding variables (study duration, attention, and word 
frequency) were unsuccessful; there were no resultant effects on $\sigma_{o}$, although each manipulation was assessed to have a weak effect on recognition confidence ratings. Despite this, both $d$ and $\sigma_{\mathrm{o}}$ were found to be significantly greater in the low item characteristic variance condition in Experiment 2, suggesting again that changes in $\sigma_{\mathrm{o}}$ may result from factors other than encoding variability. Estimates of $d$ and $\sigma_{\mathrm{o}}$ also showed strong positive correlations in every experiment, indicating that old item variance may scale with mean strength. This was not predicted by the encoding variability hypothesis.

The idea that mean memory strength and variance in memory strength are related is evidenced elsewhere in the recognition memory literature. Although some previous research concluded that the $z$-ROC slope takes a constant value of approximately 0.8 (Ratcliff, Sheu, \& Gronlund, 1992; Ratcliff, McKoon, \& Tindall, 1994), it was later found that in many cases, increases in mean strength generally decrease the $z$-ROC slope (Glanzer et al., 1999; Parks \& Yonelinas, 2007), meaning that mean strength and old item variance increase with one another in several experimental contexts. The finding that greater strength coincides with greater old item variance has since been observed in other studies (Glanzer \& Adams, 1990; Heathcote, 2003; Hirshman \& Hostetter, 2000; Koen et al., 2013; but see Starns, Ratcliff \& McKoon, 2012; Grider \& Malmberg, 2008). More recently, Dopkins, Varner, and Hoyer (2017) found that a semantic priming manipulation increased the memory strength of new items and the variance of their corresponding confidence ratings at test, as well as the $z$-ROC slope. This suggests that a form of strength and item variance scaling could apply more generally to both old and new item types - a distribution with a greater mean tends to have a greater variance. In sum, this is evidence that $\sigma_{\mathrm{o}}$ scales as a monotonically increasing function of $d$ in many experimental settings.

Our first two experiments aim to test whether estimates of $\sigma_{\mathrm{o}}$ are affected by encoding variability or mean memory strength. To achieve this, a successful manipulation of encoding 
variability during the study phase is needed. Despite previous efforts by Spanton and Berry (2020) to add Gaussian variability to individual item characteristics, the resultant effects upon old item variance were weak. This may be because even without experimental manipulation, there are already a very large number of encoding variables that sum to determine levels of added strength in any condition. Therefore, any further attempts to experimentally manipulate a given encoding variable might have a minimal effect on old item variance because added strength already varies greatly. It could also be possible that the effect of any experimentally manipulated encoding variable is partially counteracted by any number of other encoding variables that occur naturally. When manipulating item characteristics for example, if word frequency and strength are negatively related whereas concreteness and strength are positively related, then any amount of added strength that a word may receive for having low word frequency may be balanced by a decrement in strength if that word also happens to have low concreteness. Furthermore, there is likely to be a negative correlation between an item's baseline strength value and the increment of added strength it receives during study (Jang et al., 2012), which, in conjunction with the aforementioned factors, could make it difficult to establish a strong experimental manipulation of encoding variability (Spanton \& Berry, 2020).

A potential way to address these problems is to manipulate multiple item characteristics simultaneously to achieve a greater combined experimental effect upon old item variance. In doing so, the possibility that manipulated item characteristics may systematically counteract each other can also be addressed by ensuring that these characteristics are correlated within a word list. Returning to the example above, word frequency and concreteness would be less likely to counteract one another if their values were negatively correlated, increasing their summated effect upon the variance of recognition confidence judgements. Such a condition could be compared with another wherein item characteristics are constrained to be as low in variance as 
possible, resulting in low encoding variability. Furthermore, if the mean of each item characteristic measure is equal across word lists in both high and low variability conditions, the overall memorability of stimuli in each set would be controlled. This control of overall stimulus memorability within an item characteristic manipulation allows for memory strength to be manipulated orthogonally as a separate factor.

Our third experiment attempts to test the encoding variability hypothesis by including low and high item characteristic variance stimulus conditions within a single test phase, rather than separate ones. In each condition of Experiments 1 and 2, the characteristics of old and new items had approximately equal variance. This prevents some words in high encoding variability lists being artefactually more discriminable based on their extreme characteristics, which would confound the orthogonal manipulation of mean memory strength. However, as $\sigma_{\mathrm{o}}$ is conceptualized as the ratio of new/old item variance in the UVSD model, it is possible that our item characteristic manipulations would not affect this parameter, unless old items gain added variability purely by virtue of being studied (Wixted, 2007). Experiment 3 addresses this possibility to provide a new test of the encoding variability hypothesis. Experiments 1 and 2 were preregistered on the Open Science Framework (https://osf.io/ty8vz/), with details of our main hypotheses, experimental designs, methods, and analyses being disclosed before data collection for each respective experiment. Deviations from our preregistration were also disclosed. Materials, data, and analyses from Experiment 3 are also found in our OSF repository.

\section{Experiment 1}

In the following experiment, we manipulate both variability in item characteristics and memory strength at two levels each (high, low) in a $2 \times 2$ factorial design. Strength will be manipulated using a one-back digit judgement task identical to that in the 'fixed' condition in 
Experiment 2 of Spanton and Berry (2020). This task will be present as a simultaneous distraction in low strength condition study phases and absent in high strength conditions. In high variability conditions, words will be selected in a manner that attempts to ensure that they have high Gaussian variance in terms of four normalized variables previously shown to influence memory strength: 1) word frequency, which was shown to have significant effects on various recognition memory accuracy metrics in multiple studies (Glanzer \& Bowles, 1976), 2) concreteness, shown to have a roughly $8 \%$ effect on correct recognition rate by Fliessbach, Weis, Klaver, Elger, and Weber (2006), 3) age of acquisition (AOA; Cortese, Khanna \& Hacker, 2010), shown to have a weak-moderate association with recognition confidence ratings, and 4) word length, which was shown to have a moderate negative relationship with correct recognition rate (Cortese, McCarty \& Schock, 2010; Cortese et al., 2010).

Besides word length, each variable will be inter-correlated to promote maximal effects upon recognition confidence ratings. In contrast, words in low variability conditions will have low variance in terms of the above variables (and a fixed word length), with mean word frequency, concreteness, and AOA scores equal to those in high variability conditions. After fitting the UVSD model to the data, we expect a main effect of our strength manipulation on $d$, with no main effect of item characteristic variability on $d$, and no interaction. Given this outcome, if mean memory strength influences old item variance, we expect a main effect of strength on $\sigma_{o}$, with no main effect of item characteristic variability and no interaction. In contrast, if the encoding variability hypothesis holds (and is represented by our manipulation of item characteristics), we would expect a main effect of item characteristic variability on $\sigma_{o}$ with no main effect of strength and no interaction. 


\section{Methods}

\section{Participants}

64 participants (12 males, 52 females) with a mean age of $22.30(S D=8.78)$ from the University of Plymouth Psychology Participation Pool took part in this experiment. Each participant was a University of Plymouth psychology undergraduate, fluent in English as a first language and not dyslexic. Participants received course credits or $£ 8$ cash payment for their participation. We justified our sample size on the basis that it was compatible with our partial counterbalancing design (see Design and Procedure), and that it gave us sufficient power to detect a small-medium effect size (i.e., Cohen's $f(V)=.36$, with $\alpha=.05$ and .80 power in a $2 \times 2$ within-subjects ANOVA). This experiment, along with the others in this article, was conducted with ethical approval from the University of Plymouth Faculty of Health Ethics Committee.

\section{Materials}

A total of 480 unique words were used as stimuli (60 old and 60 new in each condition). Chosen words appeared in the SUBTLEX-UK word database (Van Heuven, Mandera, Keuleers, \& Brysbaert, 2014) and databases from Brysbaert, Warriner, and Kuperman (2014) and Kuperman, Stadthagen-Gonzalez, and Brysbaert (2012). Names, proper nouns, and hyphenated words were excluded from an aggregate of the above databases before sampling. Word frequency scores for these words were taken from the SUBTLEX-UK database (Van-Heuven et al., 2014), concreteness scores were taken from Brysbaert et al. (2014), and AOA scores were taken from Kuperman et al. (2012). In high item characteristic variability conditions, each set of old or new words (four in total) was selected using an algorithm with the following criteria:

1. Words must be $4-10$ characters long.

2. Each set of words must have approximately equal mean word frequency $(\sim 3)$, concreteness $(\sim 3)$, and AOA ( 10) scores (see Table 1 for exact values). 
3. Concreteness and AOA scores must be strongly negatively correlated with word frequency scores within each word list $(r<-.77$ for concreteness and word frequency scores, $r<-.61$ for AOA and word frequency scores, and $r>.26$ for concreteness and AOA scores).

4. The distribution of word frequency, concreteness and AOA scores must not significantly deviate from a normal within each set, according to an Anderson-Darling test $(p>.05)$.

The remaining four sets of old/new words in the low item characteristic variability condition were sampled with the following criteria:

1. Words must be 7 characters long.

2. Each set of words must have approximately equal mean word frequency, concreteness, and AOA scores (with the same constraints as the high item characteristic variance condition).

3. Each item characteristic variable must not be highly correlated. Among the word lists generated, word frequency and concreteness had a maximum negative correlation of $r=-$ .36. Word frequency and AOA had a maximum negative correlation of $r=-.11$. Concreteness and AOA had a maximum positive correlation of $r=.03$.

4. Word frequency, concreteness and AOA scores must have low variance. For each word, the formula $\Sigma\left|\left(\mu_{e}-e_{i}\right)\right|$ was used to determine the summed difference between the mean of each item characteristic (e) across all possible words, and its corresponding value in the $i^{\text {th }}$ word. The 240 words with the lowest summed difference scores were then randomly sampled from without replacement to create the low encoding variability word lists.

In low strength conditions, participants heard audio clips of a female computer-generated voice speaking a number between 1 and 9 in each trial; this audio was absent in high strength conditions. The whole experiment was conducted on Lenovo desktop computers running an OpenSesame program (Mathôt, Schreij, \& Theeuwes, 2012) which displayed all stimuli, instructions, and logged response data. Stimuli were presented in 40 pt 'mono' font. 
Table 1.

Mean word frequency, concreteness, and age of acquisition scores in Experiments 1 and 2, with standard deviations in brackets.

\begin{tabular}{llcccc} 
& & \multicolumn{2}{c}{ Experiment 1} & \multicolumn{2}{c}{ Experiment 2} \\
\cline { 3 - 6 } Encoding Variable & Word List & $\begin{array}{c}\text { High } \\
\text { Variability }\end{array}$ & $\begin{array}{c}\text { Low } \\
\text { Variability }\end{array}$ & $\begin{array}{c}\text { High } \\
\text { Variability }\end{array}$ & $\begin{array}{c}\text { Low } \\
\text { Variability }\end{array}$ \\
\cline { 3 - 6 } Word Frequency & 1 & $2.94(0.94)$ & $2.95(0.31)$ & $2.94(1.21)$ & $2.99(0.38)$ \\
& 2 & $2.88(0.92)$ & $2.92(0.36)$ & $2.95(1.26)$ & $2.97(0.37)$ \\
Concreteness & 3 & $2.87(0.95)$ & $2.97(0.33)$ & $2.89(1.22)$ & $2.94(0.34)$ \\
& 4 & $2.89(0.93)$ & $2.94(0.32)$ & $2.95(1.26)$ & $2.90(0.37)$ \\
& 1 & & & & \\
Age of Acquisition & 1 & $3.10(0.87)$ & $3.14(0.44)$ & $3.10(1.14)$ & $3.07(0.36)$ \\
& 3 & $3.09(0.87)$ & $3.07(0.51)$ & $3.12(1.08)$ & $3.18(0.49)$ \\
& 4 & $3.09(0.87)$ & $3.17(0.51)$ & $3.11(1.21)$ & $3.11(0.48)$ \\
& 2 & $3.05(0.82)$ & $3.09(0.41)$ & $3.07(1.12)$ & $3.14(0.47)$ \\
& 3 & $10.30(2.42)$ & $10.40(0.56)$ & $10.40(3.33)$ & $10.30(0.53)$ \\
& 4 & $10.30(2.31)$ & $10.40(0.51)$ & $10.40(2.98)$ & $10.30(0.49)$ \\
& $10.30(2.52)$ & $10.40(0.48)$ & $10.40(3.40)$ & $10.40(0.58)$ \\
& $10.40(2.25)$ & $10.20(0.56)$ & $10.20(3.69)$ & $10.30(0.44)$ \\
\hline
\end{tabular}

\section{Procedure}

Participants completed all four experimental conditions in a within-subjects design. The order of conditions, the order of high item characteristic variability word lists, and the order of low item characteristic variability word lists were all partially counterbalanced according to a Latin square. This resulted in a $4 \times 4 \times 4$ partial counterbalancing design. All participants gave informed consent using a keypress response.

Before participants began their first low strength condition, they completed practice trials where they responded to auditory distractor digits without having to remember items simultaneously. In these practice trials, a fixation point was presented for $500 \mathrm{~ms}$, followed by an auditory digit and a simultaneous visual prompt to respond to the digit from the previous trial. 
This prompt appeared in the centre of the screen, lasting $3000 \mathrm{~ms}$ (on the first practice trial, participants were prompted to make no response as there was no previous trial). This was followed by a $500 \mathrm{~ms}$ inter-trial interval (ITI), during which no information was presented in the centre of the screen. The key " $\mathrm{Z}=$ Previous number even, $\mathrm{M}=$ Previous number odd" remained static near the bottom of the screen for the duration of the practice trials; participants made responses when prompted using the $\mathrm{Z}$ and $\mathrm{M}$ keys as instructed. To advance to the following study phase, participants had to make eight consecutive correct responses; if they did not do so after 30 trials, the experimenter would re-explain the task to the participant before they attempted the practice trials again.

In each condition of the main experiment, participants then completed a 60-trial study phase. The low strength study phases shared the same trial level procedure as the practice phase, with the exception that instead of a prompt to respond to the previous number, a randomly selected old word was presented in the centre of the screen. In high strength conditions, participants did not have to complete a simultaneous one-back task. Features associated with this task were therefore not present in these conditions, such as the auditory digits and the response key, although the duration of the fixation, stimulus presentation and ITI remained the same. In all conditions, participants were instructed to try their best to pay consistent attention to each word during study.

In between study and test phases in each condition, participants completed a short retention interval in which they answered basic arithmetic questions. These questions took the form " $A \pm B \pm C=$ ?" where $A, B$, and $C$ were one or two digit positive integers. The correct answer was always a one or two digit integer. Participants completed sequential trials of these questions for 60 seconds, at which point they progressed to the test phase. 
In every condition, test phases were identically structured; a fixation point would appear for $500 \mathrm{~ms}$, followed by a randomly selected word that was either old or new in the centre of the screen. This word was presented until the participant made a recognition confidence judgement based on their degree of certainty that the item was old or new. Participants made these responses with 1-6 keys at the top of the keyboard, using the category scale " 1 - Sure New, 2 - Probably New, 3 - Guess New, 4 - Guess Old, 5 - Probably Old, 6 - Sure Old”. This key, and the prompt "New or Old?" were presented near the bottom of the screen as a static reminder of the response categories throughout each test phase. After each response, a $500 \mathrm{~ms}$ ITI (in which no information was displayed in the centre of the screen) was displayed, before the next trial. Participants were instructed to make use of the whole rating scale, and to prioritize the accuracy of their judgements over speed as they completed the task.

\section{Results}

All analyses were conducted in the statistical programming language $\mathrm{R}$ (Version 4.2.0; $\mathrm{R}$ Core Team, 2022), primarily using the tidyverse package (Wickham et al., 2019). All Bayes Factors (Scaled JZS) were reported using the BayesFactor package (Morey \& Rouder, 2018). The UVSD model was fit to the data using maximum likelihood estimation (Dunn, 2010).

In the following analyses we excluded four participants who predominantly used the "Sure New" and "Sure Old" responses, resulting in large outlying parameter estimates (over 3 standard deviations above the mean estimates for $\sigma_{\mathrm{o}}$ and $d$ ). We did so because these data did not give a meaningful representation of variability in recognition responses, and because we defined this criterion for exclusion in our preregistration. We also analyzed the natural logarithmic 
transformation of $\sigma_{\mathrm{o}}$ because, with the value of $\sigma_{\mathrm{n}}$ fixed to equal $1, \sigma_{\mathrm{o}}$ is a ratio and would otherwise violate the assumptions of a $2 \times 2$ ANOVA.

\section{Study Task Performance}

The proportions of correct responses made in each 'low strength' study phase condition were compared to check whether the presence of an item characteristic variability manipulation resulted in any task interference effects. The mean proportion of correct one-back task responses did not differ significantly between the "Low Strength, High Variability" condition $(M=.94, S E$ $=.01)$ and "Low Strength, Low Variability" condition $(M=.94, S E=.01), t(59)=0.34, p=.74$, $95 \%$ CI $[-0.02,0.02], \mathrm{BF}=0.15$.

\section{Item Characteristic Variability Manipulation}

To confirm whether our manipulation of item characteristic variability influenced subsequent recognition ratings, multiple regression analyses were conducted within each condition for each participant. Word Frequency, Concreteness, Age of Acquisition and Word Length were specified as predictors of recognition confidence ratings for each old item at test. The proportion of significant regression models (as assessed by the $F$-statistic) and mean $R^{2}$ values for each condition are reported in Table 2.

To compare these $R^{2}$ values, we conducted a $2 \times 2$ ANOVA on $R^{2}$ with strength (high, low) and item characteristic variability (high, low) as factors. There was no main effect of strength on $R^{2}, F(1,59)=0.45, p=.51, \eta_{\mathrm{p}}^{2}=.01, \mathrm{BF}=0.17$. However, there was a significant effect of item characteristic variability on $R^{2}, F(1,63)=47.32, p<.001, \eta_{p}^{2}=.46, \mathrm{BF}=1.59 \times$ $10^{9}$, and no interaction, $F(1,59)=0.33, p=.57, \eta_{\mathrm{p}}{ }^{2}=.01, \mathrm{BF}=0.23$. This indicates that the proportion of variance in the ratings explained by the predictor variables increased because of our item characteristic manipulation and not our strength manipulation. $R^{2}$ was on average $5-6 \%$ greater in the high variability conditions than the low ones. 
Table 2.

The proportion of significant regression models and mean $R^{2}$ values (standard deviations in brackets) for each condition in Experiments 1, 2, and 3.

\begin{tabular}{|c|c|c|c|}
\hline Experiment & Condition & $P$ (significant) Regressions & Mean $R^{2}$ \\
\hline \multicolumn{4}{|c|}{ Experiment 1} \\
\hline & High Strength, High Variability & .22 & $.11(.07)$ \\
\hline & High Strength, Low Variability & .10 & $.06(.04)$ \\
\hline & Low Strength, High Variability & .23 & $.12(.07)$ \\
\hline & Low Strength, Low Variability & .03 & $.06(.04)$ \\
\hline \multicolumn{4}{|c|}{ Experiment 2} \\
\hline & High Strength, High Variability & .38 & $.13(.08)$ \\
\hline & High Strength, Low Variability & .07 & $.05(.04)$ \\
\hline & Low Strength, High Variability & .22 & $.12(.09)$ \\
\hline & Low Strength, Low Variability & .05 & $.05(.04)$ \\
\hline \multicolumn{4}{|c|}{ Experiment 3} \\
\hline & Old, High Variability & .19 & $.09(.06)$ \\
\hline & Old, Low Variability & .04 & $.05(.04)$ \\
\hline & New, High Variability & .19 & $.08(.07)$ \\
\hline & New, Low Variability & .04 & $.06(.04)$ \\
\hline
\end{tabular}

\section{Parameter Estimates}

All mean UVSD model parameter estimates for each condition are found in Table 3. To compare the influence of our manipulations upon parameter estimates of mean strength from the UVSD model, we conducted a $2 \times 2$ ANOVA on $d$ with strength (high, low) and item characteristic variability (high, low) as factors. There was a large main effect of strength manipulations on $d, F(1,59)=42.56, p<.001, \eta_{\mathrm{p}}^{2}=.42, \mathrm{BF}=2.07 \times 10^{10}$. There was no effect of item characteristic variability on $d, F(1,59)=0.63, p=.43, \eta_{\mathrm{p}}{ }^{2}=.01, \mathrm{BF}=0.16$, and no interaction was present, $F(1,59)=0.45, p=.50, \eta_{\mathrm{p}}{ }^{2}=.01, \mathrm{BF}=0.23$. 
Table 3.

Mean parameter estimates (standard deviations in brackets) output by the UVSD model, per condition, in Experiments 1 and 2. Mean values of $\sigma_{\mathrm{o}}$ were calculated using the log scale and then exponentiated.

Condition

\begin{tabular}{llcccc} 
Experiment & Parameter & $\begin{array}{c}\text { High Strength, } \\
\text { High Variability }\end{array}$ & $\begin{array}{c}\text { High Strength, } \\
\text { Low Variability }\end{array}$ & $\begin{array}{c}\text { Low Strength, } \\
\text { High Variability }\end{array}$ & $\begin{array}{c}\text { Low Strength, } \\
\text { Low Variability }\end{array}$ \\
\hline 1 & $1.50(0.76)$ & $1.42(0.79)$ & $0.92(0.57)$ & $0.92(0.71)$ \\
& $d$ & $1.43(1.32)$ & $1.36(1.33)$ & $1.27(1.29)$ & $1.28(1.27)$ \\
& $\sigma_{0}$ & $-0.94(1.13)$ & $-1.19(1.60)$ & $-1.30(1.39)$ & $-1.76(2.49)$ \\
$C_{1}$ & $-0.02(1.16)$ & $-0.13(1.12)$ & $-0.24(0.88)$ & $-0.51(1.90)$ \\
& $C_{2}$ & $0.72(0.45)$ & $0.56(0.48)$ & $0.51(0.43)$ & $0.42(0.41)$ \\
$C_{3}$ & $1.24(0.61)$ & $1.07(0.67)$ & $1.11(0.76)$ & $1.06(0.85)$ \\
$C_{4}$ & $1.90(1.04)$ & $1.77(1.06)$ & $1.93(1.05)$ & $1.86(1.21)$ \\
$C_{5}$ & & & & \\
& & $1.87(1.42)$ & $1.42(1.15)$ & $0.89(0.51)$ & $0.80(0.54)$ \\
& $d$ & $1.53(1.42)$ & $1.29(1.41)$ & $1.26(1.24)$ & $1.22(1.23)$ \\
& $\sigma_{0}$ & $-1.21(3.40)$ & $-1.04(1.86)$ & $-1.16(1.60)$ & $-1.31(1.75)$ \\
$C_{1}$ & $0.01(2.26)$ & $-0.24(1.84)$ & $-0.26(1.24)$ & $-0.31(1.31)$ \\
$C_{2}$ & $0.75(0.48)$ & $0.52(0.45)$ & $0.43(0.37)$ & $0.37(0.45)$ \\
$C_{3}$ & $1.26(0.66)$ & $1.02(0.57)$ & $0.97(0.46)$ & $0.86(0.52)$ \\
$C_{4}$ & $1.93(0.80)$ & $1.76(0.67)$ & $1.69(0.51)$ & $1.72(1.04)$ \\
\hline
\end{tabular}

The ordinal pattern of $\sigma_{\mathrm{o}}$ across conditions followed that of $d$. Another $2 \times 2$ ANOVA with strength and item characteristic variability as factors was conducted with $\sigma_{o}$ as the dependent variable. A significant main effect of strength was found, $F(1,59)=6.03, p=.017, \eta_{\mathrm{p}}{ }^{2}$ $=.10, \mathrm{BF}=6.03$. Again, there was no effect of item characteristic variability, $F(1,59)=0.49, p=$ $.49, \eta_{\mathrm{p}}^{2}=.01, \mathrm{BF}=0.20$, and no significant interaction, $F(1,59)=0.94, p=.34, \eta_{\mathrm{p}}^{2}=.02, \mathrm{BF}=$ 0.29. This is evidence that estimates of $\sigma_{\mathrm{o}}$ were determined by mean memory strength, rather than encoding variability from our manipulated item characteristics. 
Table 4.

Best fitting regression models relating mean strength and old item variance in each experiment, with $R^{2}$ values.

\begin{tabular}{llll} 
Experiment & Condition & Best Fitting Model & $R^{2}$ \\
\hline Experiment 1 & & & .23 \\
& High Strength, High Variability & $\sigma_{\mathrm{o}}=0.09+0.18(d)$ & .24 \\
& High Strength, Low Variability & $\sigma_{\mathrm{o}}=0.05+0.18(d)$ & .28 \\
& Low Strength, High Variability & $\sigma_{\mathrm{o}}=0.02+0.23(d)$ & .26 \\
& Low Strength, Low Variability & $\sigma_{\mathrm{o}}=0.09+0.17(d)$ & .56 \\
Experiment 2 & & & .50 \\
& High Strength, High Variability & $\sigma_{\mathrm{o}}=0.08+0.18(d)$ & .20 \\
& High Strength, Low Variability & $\sigma_{\mathrm{o}}=-0.04+0.21(d)$ & .09 \\
& Low Strength, High Variability & $\sigma_{\mathrm{o}}=0.06+0.19(d)$ & .32 \\
& Low Strength, Low Variability & $\sigma_{\mathrm{o}}=0.11+0.11(d)$ & .23 \\
\hline & & & \\
& Old, High Variability & $\sigma_{\mathrm{oh}}=0.01+0.23(d)$ & \\
& Old, Low Variability & $\sigma_{\mathrm{ol}}=-0.01+0.19(d)$ & \\
\hline
\end{tabular}

\section{Curve-Fitting Analysis}

As an exploratory analysis, we fitted linear and polynomial models to estimates of $d$ and $\sigma_{\mathrm{o}}$ to determine the shape of the function by which $\sigma_{\mathrm{o}}$ scales with $d$. We evaluated three scaling formulae; one in which scaling is linear $\left(\sigma_{\mathrm{o}}=y+b d\right.$, where $y$ is the intercept), one with linear and quadratic components $\left(\sigma_{\mathrm{o}}=y+b_{1} d+b_{2} d^{2}\right)$, and one with linear, quadratic, and cubic components $\left(\sigma_{\mathrm{o}}=y+b_{1} d+b_{2} d^{2}+b_{3} d^{3}\right)$. In a sequential regression procedure, each model was fit to data, and the difference in the fit of each model was computed sequentially using frequentist and Bayesian ANOVAs. Linear models with intercepts between 0.04 and 0.09 and coefficients between .17 and .23 tended to fit the data best (see Table 4). In all conditions, there was no reliable improvement in fit being evident in frequentist ANOVAs from adding quadratic, or quadratic and cubic components ( $p$ s > .28). Bayesian ANOVAs also supported this conclusion (BFs < 0.44). 


\section{Discussion}

We found no evidence that varying item characteristics influenced estimates of old item variance, $\sigma_{0}$, despite our item characteristic variance manipulation having a clear impact on recognition confidence ratings. Instead, overall memory strength determined estimates of $\sigma_{\mathrm{o}}$. Moreover, curve fitting analyses showed a positive, linear association between $d$ and $\sigma_{\mathrm{o}}$, further providing evidence of an association between strength and old item variance. These results provide clear evidence that a strength scaling trend can explain the old item variance in the present experiment, with no reliable contributions of encoding variability being observed as a result of our item characteristic manipulation.

Although the effect of our item characteristic manipulation on recognition confidence ratings was significant, this effect was of small to medium size (Cohen, 1988; see Table 2 for $R^{2}$ values from each condition). It is therefore possible that, even if our manipulation was representative of encoding variability, it still might not have translated to differences in $\sigma_{\mathrm{o}}$ that were detectable. This outcome would be unable to explain the presence of the currently observed strength scaling trend; however, it would mean that the encoding variability hypothesis might also hold under a stronger manipulation. In Experiment 2, we aim to establish such a manipulation by adding even more variability to the characteristics of old items than in Experiment 1 .

\section{Experiment 2}

Although variability in item characteristics affected recognition confidence responses in Experiment 1, it is possible that the strength of this manipulation was constrained by the Gaussian distributional assumption by which item characteristic variables were sampled. This assumption was driven by the specification of the encoding variability hypothesis, which states 
that added strength is Gaussian (Jang et al., 2012). Although this assumption is plausible, the Lyapunov central limit theorem states that many non-identical independent random variables can still sum to a Gaussian form, provided they satisfy certain mathematical assumptions. In practice, it is hard to verify these assumptions since memory strength is a latent variable. However, it is possible that adding non-Gaussian strength distributions may result a product that is at least close to the Gaussian old item distribution in the UVSD. To this end, Experiment 2 will follow a method similar to Experiment 1, although the distributions of item characteristic values will be permitted to be non-Gaussian. This will maximize the variability of item characteristics even more than in Experiment 1, thereby increasing the chance of a detectable effect of encoding variability. If this manipulation is successful, the same predicted outcomes from Experiment 1 apply.

\section{Methods}

\section{Participants}

64 participants (16 males, 47 females) with a mean age of $22.8(S D=10.7)$ from the University of Plymouth Psychology Participation Pool took part in this experiment in exchange for either $£ 8$ or course participation points. Each participant spoke English fluently as a first language, was not dyslexic, and had not participated in Experiment 1. Participants were either University of Plymouth psychology undergraduates, or members of the public from the Plymouth area.

\section{Materials and Procedure}

Stimuli were 480 words (60 old and 60 new in each condition). These words were sampled with the same constraints as in the previous experiment, with only the following differences: 
1. The requirement for the distributions of word frequency, concreteness, and AOA scores to not significantly deviate from a Gaussian in the high encoding variability lists was removed. Instead, the distributions did not strictly adhere to any preset distributional shape and were only constrained to be roughly symmetrical. This was achieved by scoring each word by a weighted index of word frequency, concreteness, and AOA scores, and grouping words based on their distance from the mean of the index, measured in standard deviations. Words were then randomly sampled in equal quantities from each group, resulting in distributions of each encoding variable that were non-Gaussian and had more variance than in Experiment 1.

2. Due to the sampling method, the correlations between item characteristics were stronger, despite no threshold correlation values being imposed as generative constraints. The negative correlations between word frequency and concreteness ranged between $r=-.89$ and $r=.92$. The negative correlations between word frequency and AOA were between $r=-.60$ and $r=-.77$. The positive correlations between concreteness and AOA were between $r=.50$ and $r=.65$.

The strength manipulation and other materials were identical to the previous experiment. The procedure was also identical to that of Experiment 1, with the only difference being that new word lists replaced those that were previously used. 


\section{Results}

We excluded four participants who used the "Sure New" and "Sure Old" responses in nearly all test phase trials. These exclusions were made for the same reasons as those in Experiment 1 . We also analyzed the natural logarithm of $\sigma_{o}$, as in the previous experiment.

\section{Study Task Performance}

As in Experiment 1, the mean proportion of correct responses in the "Low Strength, High Variability" condition $(M=.92, S E=.01)$ was not significantly different from that in the "Low Strength, Low Variability" condition $(M=.93, S E=.01), t(59)=-0.31, p=.76,95 \%$ CI $[-0.04$, $0.03], \mathrm{BF}=0.15$.

\section{Item Characteristic Variability Manipulation}

Our item characteristic variability manipulation was assessed using the same multiple regression analysis as in Experiment 1. The proportion of significant regression models and mean $R^{2}$ values for each condition are reported in Table 2 . A $2 \times 2$ ANOVA on $R^{2}$ with strength and item characteristic variability as factors found no main effect of strength on $R^{2}, F(1,59)=0.79, p$ $=.38, \eta_{\mathrm{p}}{ }^{2}=.01, \mathrm{BF}=0.17$. There was, however, a significant effect of item characteristics on $R^{2}$, $F(1,59)=45.21, p<.001, \eta_{\mathrm{p}}^{2}=.43, \mathrm{BF}=5.37 \times 10^{12}$ and no interaction, $F(1,59)=0.61, p=$ $.44, \eta_{\mathrm{p}}^{2}=.01, \mathrm{BF}=0.24$. As in Experiment 1, this indicates that the proportion of variance in recognition confidence ratings accounted for by the predictor variables increased between $7-8 \%$ with our item characteristic variability manipulations, and not our strength manipulation.

\section{Parameter Estimates}

Mean parameter estimates for Experiment 2 are presented in Table 3. $2 \times 2$ ANOVAs were conducted to determine whether our variability or strength manipulations influenced estimates of $d$. There was a significant main effect of strength on $d, F(1,59)=41.96, p<.001$, $\eta_{\mathrm{p}}^{2}=.42, \mathrm{BF}=6.68 \times 10^{9}$. There was also a significant main effect of item characteristic 
variability, although this was accompanied by an inconclusive Bayes Factor, $F(1,59)=9.98, p=$ $.003, \eta_{\mathrm{p}}^{2}=.15, \mathrm{BF}=1.63$. There was not a significant interaction, $F(1,59)=3.70, p=.06, \eta_{\mathrm{p}}^{2}=$ $.06, \mathrm{BF}=0.79$. Our strength manipulations were therefore shown to have a main effect on $d$, however, there was some weak evidence of an effect of item characteristic variability as well. This suggests that our non-Gaussian item characteristic manipulation may have had some unintended effect upon memory strength.

To assess whether variability in item characteristics or overall strength influenced estimates of $\sigma_{0}$, we conducted a $2 \times 2$ ANOVA. There was a significant main effect of strength on $\sigma_{\mathrm{o}}, F(1,59)=11.99, p=.001, \eta_{\mathrm{p}}{ }^{2}=.17, \mathrm{BF}=46.77$. There was also a significant effect of item characteristic variability on $\sigma_{\mathrm{o}}, F(1,59)=11.16, p=.001, \eta_{\mathrm{p}}{ }^{2}=.16, \mathrm{BF}=7.00$. There was also no significant interaction, $F(1,59)=3.22, p=.08, \eta_{\mathrm{p}}{ }^{2}=.05, \mathrm{BF}=1.22$. In sum, there was strong evidence for both an effect of strength and item characteristic variability on $\sigma_{\mathrm{o}}$.

\section{Curve-Fitting Analyses}

We conducted the same curve-fitting analyses as in the previous experiment; results from this analysis are found in Table 4. Linear models fitted best in all conditions, as quadratic and cubic components did not improve model fit $(p s>.18, \mathrm{BFs}<0.70)$.

\section{Discussion}

Unlike in Experiment 1, there was evidence for main effects of both item characteristics and overall strength on estimates of $\sigma_{\mathrm{o}}$ in Experiment 2. However, contrary to the aims of our study, our manipulation of item characteristic variability significantly affected estimates of $d$, though the Bayes Factor for this result was inconclusive. It is therefore difficult to judge whether some effect of our item characteristic manipulation on $\sigma_{\mathrm{o}}$ was the genuine result of increased encoding variability, or a consequence of the manipulation also affecting memory strength. What 
is clearer is that our manipulation of memory strength influenced both estimates of $d$ and $\sigma_{\mathrm{o}}$, and that this is not explicitly accounted for by the current specification of the encoding variability hypothesis.

It is possible that our non-Gaussian item characteristic variability manipulation gave rise to the unexpected effects of item characteristic variability on $d$. Although we aimed to sample words with roughly symmetrical distributions of word frequency, concreteness, and AOA values, it is possible that deviating from a Gaussian form caused the distributions of these item characteristics to be less symmetrical than those in Experiment 1. This could have resulted in our manipulation having unintended effects on old item memory strength, shifting the value of $d$ as well as affecting $\sigma_{0}$. Indeed, our variability manipulation in Experiment 1 did not have unexpected effects on $d$ as well as $\sigma_{\mathrm{o}}$, despite the only major difference between each experiment being the distributional assumption by which words were sampled. In any case, it is still more certain that overall memory strength has a substantial effect on estimates of $\sigma_{\mathrm{o}}$ than our manipulation of item characteristics in this experiment.

\section{Experiment 3}

In our present methods (and those in Spanton \& Berry, 2020), we matched the level of manipulated variability in item characteristics across the old and new word lists in a test phase. Specifically, in Experiments 1 and 2, the old and new words in each test phase had very similar high or low variability in terms of word frequency, concreteness, and AOA. This decision was made to minimize the chance that participants would use differences in item characteristics in each list as an additional memory cue, which would confound our manipulations. We assumed that in these methods, $\sigma_{\mathrm{o}}$ would still be greater in high variability conditions if the encoding variability hypotheses were to be true. However, it is important to note that $\sigma_{\mathrm{o}}$ is a ratio of target- 
lure variance. This is the case regardless of the fixed value of $\sigma_{\mathrm{n}}$, although we fixed it to equal 1 to ensure the target-lure ratio and the absolute value of $\sigma_{\mathrm{o}}$ were equal. Consequently, if our manipulation of item characteristic variance affected $\sigma_{\mathrm{n}}$, it is possible that in our previous experiments, any effect of old item characteristic variability on $\sigma_{0}$ may have been offset by the high, matched level of variance of new item strength. This would lower the chance of observing an encoding variability effect.

To circumvent this issue while mitigating the possibility that item characteristics in each old or new word list might serve as an additional memory cue, we can design an experiment with a single study/test phase. Within this phase, half of the old and new items can have highly variable item characteristics (word frequency, concreteness, AOA, and word length), whereas the other conditions can have low variance in their item characteristics. Overall memory performance and variability in memory for these four stimulus conditions ("old-high", "old-low", "new-high", "new-low") can be analyzed separately. However, because the complete old and new word lists share the same overall variability in item characteristics, participants cannot use differences in these characteristics as a cue to aid their recognition judgements. If the variance in memory strength for each condition is modelled on a participant level, the encoding variability hypothesis would predict that this variance would be greater in the old-high condition $\left(\sigma_{\mathrm{oh}}\right)$ than in the oldlow condition $\left(\sigma_{\mathrm{ol}}\right)$.

To allow the estimation of the key parameters in this experiment, we must define four distributions in the UVSD model - one for each condition (see Figure 1). The mean and standard deviation of the new-low distribution can be fixed so that $\mu_{\mathrm{nl}}=0$ and $\sigma_{\mathrm{nl}}=1$, allowing the means and standard deviations of each other condition to be free and scaled upon these fixed parameters. Since all the conditions appear to the participant in one study-test phase, it follows that the same decision criteria should be used to model judgements for words in every sub-list. Extending the 
UVSD model to represent this design therefore requires the free parameters $\theta=\left\{\mu_{\mathrm{nh}}, \mu_{\mathrm{ol}}, \mu_{\mathrm{oh}}, \sigma_{\mathrm{nh}}\right.$, $\left.\sigma_{\mathrm{ol}}, \sigma_{\mathrm{oh}}, C_{1}, C_{2}, \ldots C_{I}\right\}$. The specification of this model extension, alongside its likelihood function and parameter recovery simulations, can be found in Appendix A.

Figure 1.

A depiction of our extended UVSD model specification, with parameters set to the mean estimates recovered from Experiment 3.

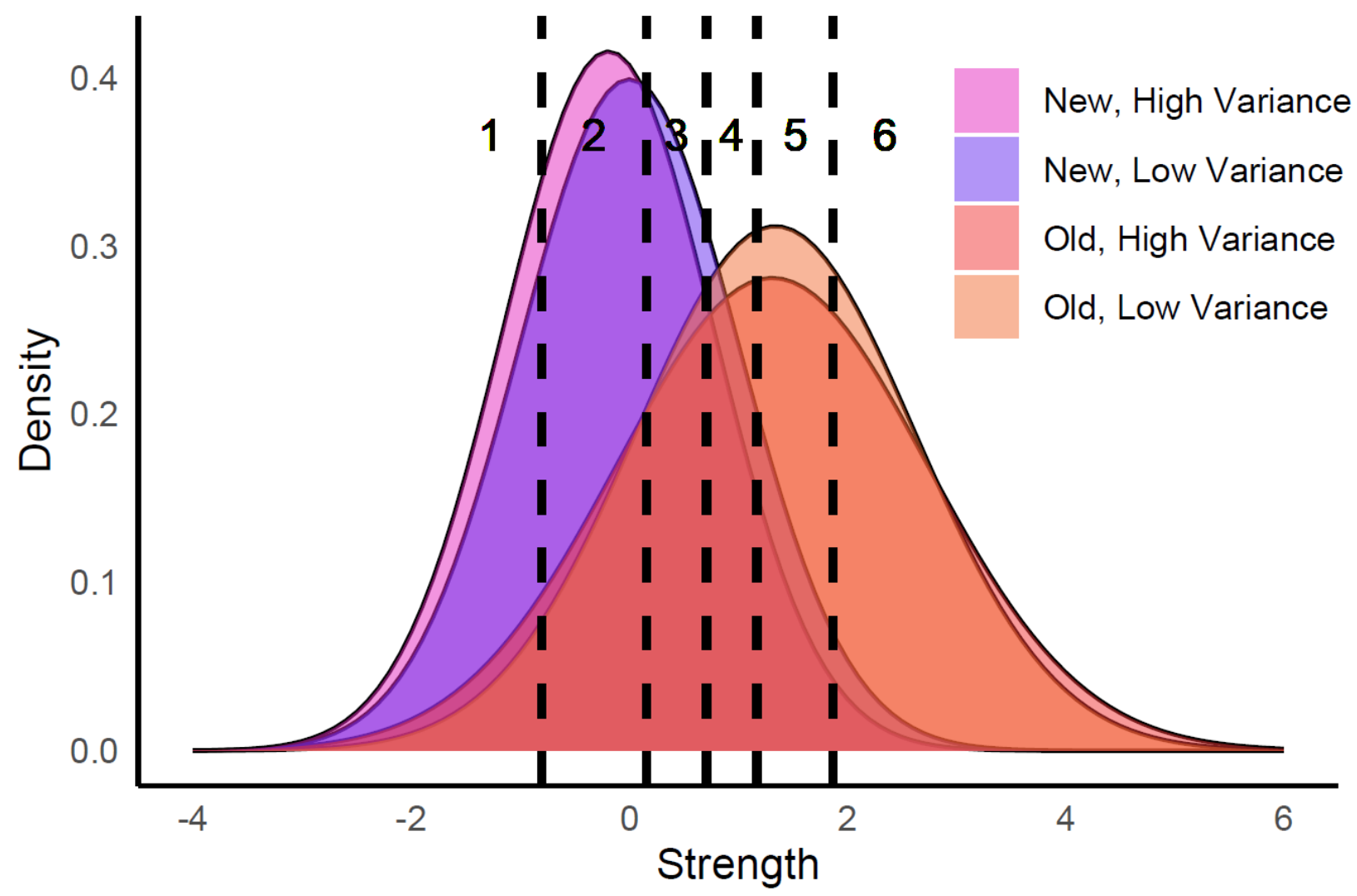

\section{Method}

\section{Participants}

75 undergraduate psychology students from the University of Plymouth (57 females, 16 males, 2 non-binary/other) completed the experiment in exchange for course credits. Three 
participants were excluded during analyses due to outlying parameter estimates (see Results), leaving an effective sample of 72 participants that allowed for detection of a minimum effect size $d_{\mathrm{z}}=0.33$ at $80 \%$ power in a paired samples $t$-test.

\section{Materials}

The stimuli consisted of the first two lists of high variability words and low variability words from Experiment 1. The experiment was implemented using the OSWeb functionality of OpenSesame (Mathôt et al., 2012). Participants completed the task in a lab, using Lenovo desktop computers running a browser window containing the experiment program.

\section{Procedure}

Participants first completed a study phase consisting of 120 trials. In each trial, they viewed a fixation point for $500 \mathrm{~ms}$, a word for $3000 \mathrm{~ms}$, and an inter-trial interval (a blank screen) for $500 \mathrm{~ms}$. The words in the study phase were made up of one set of 60 high variability words, and one set of 60 low variability words; these sets were intermixed and presented in a different random order for each participant. The allocation of each high and low variability word list as old or new item was also randomized across participants. Participants were instructed to pay attention to each word during the study phase, and that they should try to remember as many words as possible for a later memory test. After the study phase, participants had a 60 second break before reading instructions for the test phase.

The test phase had the same trial level structure as those in Experiments 1 and 2, with participants making recognition judgements on the same 1-6 scale. A total of 240 words were presented (120 old, 120 new), with the new words consisting of the remaining high and low item variability lists. As in the study phase, each participant completed a different random order of trials. Upon completing the test phase, participants input their age and gender into the experimental program before reading a full debrief. 


\section{Results}

Three participants were excluded from all analyses for having outlying parameter estimates, in line with the approach taken in Experiments 1 and 2. We also log transformed the parameters $\sigma_{\mathrm{oh}}, \sigma_{\mathrm{ol}}$, and $\sigma_{\mathrm{nh}}$ in line with our approach in Experiments 1 and 2 . Bonferroni corrections were applied to all pairwise comparisons.

\section{Item Characteristic Variability Manipulation}

As in Experiments 1 and 2, regression analyses were conducted to gauge the effect of each manipulated item characteristic on recognition confidence responses. Each participant's data was split by item type (old, new) and item characteristic variability level (high, low), and regression models with word frequency, concreteness, AOA, and word length as predictors were fit to each combination of factors. The proportion of significant regression models and mean $R^{2}$ values can be found in Table 2 . A $2 \times 2$ within subjects ANOVA on $R^{2}$ was then conducted with item type and item characteristic variability level as factors. This ANOVA revealed a significant main effect of item characteristic variability on $R^{2}, F(1,71)=18.67, p<.001, \eta_{\mathrm{p}}{ }^{2}=.21, \mathrm{BF}=1018.28$. There was no significant main effect of item type, $F(1,71)=0.27, p=.61, \eta_{\mathrm{p}}{ }^{2}<.01, \mathrm{BF}=0.15$, and no significant interaction, $F(1,71)=2.33, p=.13, \eta_{\mathrm{p}}{ }^{2}=.03, \mathrm{BF}=0.50$. This indicates that variance in recognition confidence ratings was explained by our item characteristic variability manipulation, rather than the presence of words in the study phase. The high variability words accounted for around $4 \%$ more total variance in recognition confidence ratings for old items than low variability words, which is a roughly comparable increase with Experiments 1 and 2. 
Table 5 .

Mean parameter estimates for the UVSD model in Experiment 3 (standard deviations in brackets).

\begin{tabular}{lll} 
Parameter & $M$ & $S D$ \\
\hline$\mu_{\mathrm{nh}}$ & -0.19 & $(0.28)$ \\
$\mu_{\mathrm{ol}}$ & 1.34 & $(0.69)$ \\
$\mu_{\mathrm{oh}}$ & 1.31 & $(0.77)$ \\
$\sigma_{\mathrm{nh}}$ & 0.96 & $(1.36)$ \\
$\sigma_{\mathrm{ol}}$ & 1.28 & $(1.31)$ \\
$\sigma_{\mathrm{oh}}$ & 1.42 & $(1.40)$ \\
$C_{1}$ & -0.80 & $(1.22)$ \\
$C_{2}$ & 0.16 & $(0.60)$ \\
$C_{3}$ & 0.71 & $(0.44)$ \\
$C_{4}$ & 1.17 & $(0.56)$ \\
$C_{5}$ & 1.87 & $(0.70)$ \\
\hline
\end{tabular}

Note: The fixed parameters are not shown here: $\mu_{\mathrm{nl}}$ was fixed to 0 , and $\sigma_{\mathrm{nl}}$ was fixed to 1 . The mean and standard deviation of each log-transformed sigma parameter was calculated, then exponentiated.

\section{Parameter Estimates}

The parameter estimates from the UVSD model can be found in Table 5. A one-factor repeated measures ANOVA with a Greenhouse-Geisser sphericity correction was used to compare the estimates of $\sigma$ in the old-high, old-low, and new-high conditions. Estimates significantly differed across conditions, $F(1.66,117.91)=85.66, p<.001, \eta_{\mathrm{p}}{ }^{2}=.55, \mathrm{BF}=1.75 \times$ $10^{10}$. The ordinal pattern of variance estimates for each distribution can be seen in Figure 2.

Pairwise comparisons confirmed that estimates of $\sigma_{\mathrm{nh}}$ were reliably lower than those of both $\sigma_{\mathrm{oh}}$, $t(71)=-11.33, p<.001, \mathrm{BF}=8.27 \times 10^{14}$, and $\sigma_{\mathrm{ol}}, t(71)=-9.00, p<.001, \mathrm{BF}=2.42 \times 10^{10}$. Crucially however, estimates of $\sigma_{\mathrm{oh}}$ were significantly greater than estimates of $\sigma_{\mathrm{ol}}, t(71)=4.06$, 
$p<.001, \mathrm{BF}=300.76$. Further, a one-sample $t$-test also revealed that $\sigma_{\text {nh }}$ did not significantly differ from 1 , the fixed value of $\sigma_{\mathrm{nl}}, t(71)=-1.26, p=.21,95 \%$ CI $[-0.11,0.03]$, BF $=0.28$. This means that our manipulation of item characteristic variability only affected old items, in line with the encoding variability hypothesis.

To assess the possibility that differences in old item variance may have been driven by effects of overall memory strength, we calculated discriminability $(d)$ measures for high and low variability conditions. These measures were given by calculating $d_{\mathrm{high}}=\mu_{\mathrm{oh}}-\mu_{\mathrm{nh}}$ and $d_{\mathrm{low}}=\mu_{\mathrm{ol}}-$ $\mu_{\mathrm{nl}}$ respectively, on a participant level. Discriminability measures were reliably greater for high variability items than for low variability items, $t(71)=2.90, p=.004,95 \% \mathrm{CI}[0.05,0.28], \mathrm{BF}=$ 6.05. This increase in discriminability for high variability items was likely driven by estimates of $\mu_{\mathrm{nh}}$ being reliably lower than 0 , the fixed value of $\mu_{\mathrm{nl}}, t(71)=-5.72, p<.001,95 \%$ CI $[-0.26,-$ $0.12], \mathrm{BF}=58633.94$. By contrast, no reliable differences were found between estimates of $\mu_{\mathrm{oh}}$ and $\mu_{\mathrm{ol}}, t(71)=-0.60, p=.55,95 \% \mathrm{CI}[-0.11,0.06], \mathrm{BF}=0.15$. This means that greater overall memory strength for high variability items coincided with greater estimates of old item variance for those items. Therefore, as in Experiment 2, we cannot conclude whether increases in old item variance are due to manipulated encoding variability, because these increases were not independent of changes in overall strength.

For comparison with the results of Experiments 1 and 2, and those of Spanton and Berry (2020), we examined the relationship between discriminability and $\sigma_{\mathrm{o}}$ parameters within the high and low variability conditions using linear regression (see Table 4). There was a significant positive relationship between $\mu_{\mathrm{oh}}$ and $\sigma_{\mathrm{oh}}, F(1,70)=32.64, p<.001, R^{2}=.32$. There was also a significant positive relationship between $\mu_{\mathrm{ol}}$ and $\sigma_{\mathrm{ol}}, F(1,70)=21.07, p<.001, R^{2}=.23$. This indicates that estimates of mean memory strength and variability in memory strength for old items were positively associated. 
Figure 2.

Raincloud plots of $\sigma$ free parameter estimates and discriminability measures, with circular points denoting means. The mean of each log-sigma parameter was calculated, then exponentiated.



\section{Discussion}

The present results showed a clear increase in estimates of old item variance in high variability conditions while estimates of new item variance remained constant across high and low variability conditions. However, this selective increase in old item variance cannot be taken as clear evidence for the encoding variability hypothesis, because old and new items were also more discriminable in the high variability conditions. Therefore, as in Experiment 2, the effect of our item characteristic variability manipulation coincided with simultaneous increases in memory 
strength. Moreover, measures of discriminability and old item variance were positively associated on a participant level in both high and low variability conditions, mirroring the linear relationships found between $\sigma_{\mathrm{o}}$ and $d$ in Experiments 1 and 2.

Although we saw unexpected simultaneous effects on memory strength and old item variance in this experiment, our manipulation was at least successful in increasing old item variance while controlling new item variance. This shows that the present experimental design has promise in providing a principled test of the encoding variability hypothesis that is more certain to affect the ratio of old/new item variance than our Experiments 1 and 2. The difference in memory strength for high and low variability items in this experiment was unexpected given that the same stimuli did not by themselves elicit such a difference when used in Experiment 1. However, these results show (along with previous attempts) that it is often hard to manipulate encoding variability in a theoretically principled way without the presence of additional confounds. The present single-block design appears to be a promising way of selectively manipulating old item variance, but effects on discriminability should also be considered in future experiments with this design.

\section{General Discussion}

Although it has been suggested that encoding variability causes the old item variance effect (Wixted, 2007), previous research has suggested that it cannot solely account for the UVSD model's unequal variance assumption (Koen et al., 2013; Spanton \& Berry, 2020). Our results from Experiment 1 reiterate this conclusion, showing that $\sigma_{o}$ tends to be determined by mean strength $(d)$ in a linear scaling function, with no main effect of varying item characteristics. In Experiment 2, there was a main effect of varying item characteristics on $\sigma_{\mathrm{o}}$, though this was partially confounded by a weaker effect of item characteristic variability on $d$, and overall 
strength still had the greatest influence on $\sigma_{o}$ in this experiment. Experiment 3 showed that increasing item characteristic variability resulted in a selective increase in old item variance while new item variance remained constant. However, this once again coincided with increased discriminability measures in high variability conditions. Positive participant-level associations between old item variance and memory strength measures persisted across all experiments. We therefore conclude that overall memory strength can determine old item variance estimates in the UVSD model independently of encoding variability. By contrast, any effects of encoding variability prompted by varying item characteristics at study are not fully separable from increases in mean memory strength in the present experiments.

It has previously been stated that manipulating encoding variability by varying item characteristics would be very challenging to achieve on theoretical grounds (Spanton \& Berry, 2020). The present results reiterate this conclusion. Despite manipulating multiple item characteristics at once to achieve a meaningful effect on recognition confidence ratings - in this instance, one that causes an $R^{2}$ difference between conditions corresponding to a small to medium effect size (Cohen, 1988) - this did not lead to clear increases in old item variance. Moreover, it proved difficult to manipulate mean memory strength orthogonally without confounds, as seen in Experiment 2. In Experiment 3, we were able to contribute a new manipulation that affected the old/new item variance ratio but were unable to control mean memory strength to observe an unequivocal encoding variability effect, despite using stimuli from Experiment 1 that did not previously elicit confounding effects on mean strength. In sum, these experiments emphasise that the encoding variability hypothesis is difficult to test. Further, no conclusive evidence of its independent contribution to the old item variance effect has yet been found.

Although we did not find unambiguous support for encoding variability, our results showed that mean strength manipulations can independently increase old item variance estimates 
on a group level, and that these parameter estimates have a positive linear association on a participant level. These findings align with previous reports of the $z$-ROC slope decreasing as memory performance increases (Glanzer et al., 1999; Parks \& Yonelinas, 2007), lending further support to the existence of a strength scaling trend. Based on this, it is unlikely that encoding variability alone can explain changes in old item variance in the UVSD model. It is possible that the specification of the encoding variability hypothesis suggested by Jang et al. (2012) can be extended to include strength scaling by assuming that the mean of the added strength distribution $\left(\mu_{\mathrm{A}}\right)$ scales with the variance of added strength $\left(\sigma_{\mathrm{A}}\right)$ in the equation $O=B+A$. However, this extension would not address other limitations of the encoding variability hypothesis identified by Spanton and Berry (2020). It would also not account for the effect of retrieval manipulations on estimates of old item variance in the UVSD model (Koen et al., 2013). As such, it is likely that this hybrid specification would not give a satisfactory explanation of the old item variance effect.

Although our results pertain foremost to the UVSD model, they raise the broader question of how other signal detection models and theoretical frameworks represent encoding variability and strength scaling. Despite finding no conclusive evidence for the encoding variability hypothesis specified by Jang et al. (2012) in relation to the UVSD model, we do not dispute the general idea that some items are encoded more strongly than others. This is almost certainly true, and so it is useful to consider how models that include different psychological processes or even explicit mathematical representations of how information is stored, retained, and retrieved, account for this. Doing so may provide useful information that could feed back into shaping a more valid explanation of the old item variance effect in the UVSD model. We now consider some alternative models and theoretical explanations for encoding variability and the strength scaling trend. 
There are many other signal detection models that could represent encoding variability and strength scaling. For instance, both the dual process signal detection (DPSD; Yonelinas, 1994) and mixture signal detection (DeCarlo, 2002) models can account for increases in old item variance with changes in their parameters (Spanton \& Berry, 2020). The DPSD model also predicts that an increase in the probability of recollection for old items boosts memory strength and old item variance. Without ruling out the contribution of factors during retention and retrieval, this gives a meaningful interpretation of the strength scaling trend that could be tested empirically with a manipulation of recollection. It is also worth noting that alongside these commonly used models, there are a wide variety of other possible signal detection models with different, non-Gaussian memory strength distributions (Malejka \& Broder, 2019; DeCarlo, 1998). These models could provide substantively different interpretations of trends in data due to their mathematical specifications, the implications of which should also be investigated.

Models outside of the signal detection framework that include explicit representations of items in memory could also be studied regarding encoding variability and strength scaling. Global matching models such as SAM (Gillund \& Shiffrin, 1984) and MINERVA 2 (Hintzman, 1988) predict that representations can vary due to factors at encoding, retention, and retrieval, in contrast to the encoding variability hypothesis. While these models can account for data in which old item variance scales with memory strength, both can also represent opposing trends, such as decreased variability in memory with increased performance, with plausible parameter behaviour. Due to this behaviour and the nature of their representations of feature-level memory for items, models such as these could also provide a broader theoretical perspective on the encoding variability hypothesis and the strength scaling trend.

It also stands that changes in the shape of the $z$-ROC are not exclusively caused by mnemonic factors (Malmberg \& Xu, 2006; Rabe, Lindsay \& Kliegl, 2021), and many models 
reflect this. For instance, previous models have added variability to recognition decision criteria, and have potential to add to discussion about the unequal variance assumption (Benjamin, Diaz, \& Wee, 2009). Although the addition of equal variability to all decision criteria does not affect the $z$-ROC slope (Wickelgren, 1968), it has been shown that forms of selective criterion variability can (Cabrera, Lu, \& Dosher, 2015). However, such variability may also decrease discriminability depending upon its form, putting such an effect in opposition to a scaling trend. It has also been shown that more accurate estimates of mean strength and old item variance can be obtained using models with variable criteria (Cabrera, Lu \& Dosher, 2015). Using variable criterion models may therefore inform whether strength scaling is seen in models that differentiate between decision processes and underlying mnemonic representations. Furthermore, RTCON and Diffusion models (Ratcliff \& Starns, 2009; Ratcliff \& Starns, 2013; Osth, Bora, Dennis, \& Heathcote, 2017), give lower estimates of old item variance in comparison to $z$-ROC slopes from signal detection models, prompted in part by changes in non-mnemonic sources of trial-to-trial variability. The extent to which explanations of encoding variability align with reaction time distributions from the RTCON models that support an unequal variance assumption could also be investigated in the future, with non-mnemonic factors in mind.

These models could help to establish the generality of the strength scaling trend and determine why some manipulations of strength do not change the slope of the $z$-ROC. Early work on the topic proposed that although the $z$-ROC slope is commonly less than 1 , memory strength manipulations do not affect its supposed value of $\sim 0.8$ (Ratcliff et al., 1994; Ratcliff et al., 1992). Although Glanzer et al. (1999) later presented substantial evidence demonstrating that $z$-ROC slopes are not constant, their analyses of previous studies that used stimulus repetition to manipulate memory strength found that the $z$-ROC slope consistently remained unchanged despite significant increases in strength. Similarly, although Yonelinas (1994) found a strength 
scaling trend when manipulating study list length, they found constant $z$-ROC slopes when increasing memory strength with a study time manipulation. Changes in memory performance also did not provoke changes in the z-ROC slopes in some later studies (Starns, Ratcliff \& McKoon, 2012; Grider \& Malmberg, 2008). Understanding these results while considering alternative models and theoretical explanations may provide information about the causes and boundary conditions of the strength scaling trend in Gaussian signal detection models.

To conclude, we investigated whether changes in the variance of recognition memory strength for old items in the UVSD model were prompted by manipulations of mean strength during study, or the variability of item characteristics. We found evidence that levels of overall memory strength influenced old item variance in Experiment 1, with no contribution of varying item characteristics. A main effect of overall strength was also found in Experiment 2; there was also a main effect of our item characteristic manipulation, however this was partially confounded by a simultaneous effect on memory strength. Experiment 3 again showed that increases in old item variance coincided with simultaneous increases in memory strength in an experimental design with a single study/test block. These results show that while mean memory strength can independently determine estimates of old item variance in the UVSD model, there is still no clear evidence for a contribution of encoding variability without a simultaneous increase in mean strength. We recommend the use of new theoretical perspectives to further examine these trends and their implications for the UVSD model, and for our understanding of recognition memory more broadly. 


\section{Acknowledgements}

This research was supported by the Economic and Social Research Council

(ES/N009916/1). We thank Joel Solomons for his assistance with collecting data for Experiments 1 and 2, and Jeff Starns for his comments and input towards Experiment 3.

\section{Conflict of Interest Statement}

The Authors declare that there is no conflict of interest. 


\section{References}

Benjamin, A. S., Diaz, M., \& Wee, S. (2009). Signal detection with criterion noise: Applications to recognition memory. Psychological Review, 116(1), 84-115. https://doi.org/10.1037/a0014351

Brysbaert, M., Warriner, A. B., \& Kuperman, V. (2014). Concreteness ratings for 40 thousand generally known English word lemmas. Behavior Research Methods, 46(3), 904-911. https://doi.org/10.3758/s13428-013-0403-5

Cabrera, C. A., Lu, Z.-L., \& Dosher, B. A. (2015). Separating decision and encoding noise in signal detection tasks. Psychological Review, 122(3), 429- 460. https://doi.org/10.1037/a0039348

Cohen, J. (1988). Statistical power analysis for the behavioral sciences ( $2^{\text {nd }}$ ed.). Hillsdale, NJ: Lawrence Erlbaum.

Cortese, M. J., Khanna, M. M., \& Hacker, S. (2010). Recognition memory for 2,578 monosyllabic words. Memory, 18(6), 595-609. https://doi.org/10.1080/09658211.2010.493892

Cortese, M. J., McCarty, D. P., \& Schock, J. (2015). A mega recognition memory study of 2897 disyllabic words. The Quarterly Journal of Experimental Psychology, 68(8), 1489-1501. https://doi.org/10.1080/17470218.2014.945096

DeCarlo, L. T. (1998). Signal detection theory and generalized linear models. Psychological Methods, 3(2), 186-205.

DeCarlo, L. T. (2002). Signal detection theory with finite mixture distributions: Theoretical developments with applications to recognition memory. Psychological Review, 109(4), 710-721. https://doi.org/10.1037//0033-295X.109.4.710 
Dopkins, S., Varner, K., \& Hoyer, D. (2017). Variation in the standard deviation of the lure rating distribution: Implications for estimates of recollection probability. Psychonomic Bulletin \& Review, 24(5), 1658-1664. https://doi.org/10.3758/s13423-017-1232-9

Dunn, J. C. (2010). How to fit models of recognition memory data using maximum likelihood. International Journal of Psychological Research, 3(1), 140-149. http://dx.doi.org/10.21500/20112084.859

Egan, J. P. (1958). Recognition memory and the operating characteristic. USAF Operational Applications Laboratory Technical Note.

Fliessbach, K., Weis, S., Klaver, P., Elger, C. E., \& Weber, B. (2006). The effect of word concreteness on recognition memory. NeuroImage, 32(3), 1413-1421. https://doi.org/10.1016/j.neuroimage.2006.06.007

Gillund, G., \& Shiffrin, R. M. (1984). A retrieval model for both recognition and recall. Psychological Review, 91(1), 1-67. https://doi.org/10.1037/0033-295X.91.1.1

Glanzer, M., \& Adams, J. K. (1990). The mirror effect in recognition memory: Data and theory. Journal of Experimental Psychology: Learning, Memory, and Cognition, 16(1), 5-16. https://doi.org/10.1037/0278-7393.16.1.5

Glanzer, M., \& Bowles, N. (1976). Analysis of the word-frequency effect in recognition memory. Journal of Experimental Psychology: Human Learning and Memory, 2(1), 21-31. https://doi.org/10.1037/0278-7393.2.1.21

Glanzer, M., Kim, K., Hilford, A., \& Adams, J. K. (1999). Slope of the receiver-operating characteristic in recognition memory. Journal of Experimental Psychology: Learning, Memory, and Cognition, 25(2), 500-513. https://doi.org/10.1037/0278-7393.25.2.500 
Grider, R. C., \& Malmberg, K. J. (2008). Discriminating between changes in bias and changes in accuracy for recognition memory of emotional stimuli. Memory \& cognition, 36(5), 933946. https://doi.org/10.3758/MC.36.5.933

Heathcote, A. (2003). Item recognition memory and the receiver operating characteristic. Journal of Experimental Psychology: Learning, Memory, and Cognition, 29(6), 1210-1230. https://doi.org/10.1037/0278-7393.29.6.1210

Hintzman, D. L. (1988). Judgments of frequency and recognition memory in a multiple-trace memory model. Psychological Review, 95(4), 528- 551. https://doi.org/10.1037/0033295X.95.4.528

Hirshman, E., \& Hostetter, M. (2000). Using ROC curves to test models of recognition memory: The relationship between presentation duration and slope. Memory \& Cognition, 28(2), 161-166. https://doi.org/10.3758/BF03213795

Jang, Y., Mickes, L., \& Wixted, J. T. (2012). Three tests and three corrections: Comment on Koen and Yonelinas (2010). Journal of Experimental Psychology: Learning, Memory, and Cognition, 38(2), 513-523. https://doi.org/10.1037/a0025880

Kellen, D., Klauer, K. C., \& Bröder, A. (2013). Recognition memory models and binary-response rocs: A comparison by minimum description length. Psychonomic Bulletin \& Review, 20(4), 693-719. https://doi.org/10.3758/s13423-013-0407-2

Koen, J., Aly, M., Wang, W.-C., \& Yonelinas, A. (2013). Examining the causes of memory strength variability: Recollection, attention failure, or encoding variability? Journal of Experimental Psychology: Learning, Memory, and Cognition, 39(6), 1726-1741. https://doi.org/10.1037/a0033671 
Koen, J., \& Yonelinas, A. (2010). Memory variability is due to the contribution of recollection and familiarity, not to encoding variability. Journal of Experimental Psychology: Learning, Memory, and Cognition, 36(6), 1536-1542. https://doi.org/10.1037/a0020448

Kuperman, V., Stadthagen-Gonzalez, H., \& Brysbaert, M. (2012). Age-of-acquisition ratings for 30,000 English words. Behavior Research Methods, 44(4), 978-990. https://doi.org/10.3758/s13428-012-0210-4

Malejka, S., \& Bröder, A. (2019). Exploring the shape of signal-detection distributions in individual recognition ROC data. Journal of Memory and Language, 104, 83-107.

Malmberg, K. J., \& Xu, J. (2006). The influence of averaging and noisy decision strategies on the recognition memory ROC. Psychonomic Bulletin \& Review, 13(1), 99-105. https://doi.org/10.3758/BF03193819

Mathôt, S., Schreij, D., \& Theeuwes, J. (2012). OpenSesame: An open-source, graphical experiment builder for the social sciences. Behavior Research Methods, 44(2), 314-324. https://doi.org/10.3758/s13428-011-0168-7

Morey, R. D., \& Rouder, J. N. (2018). BayesFactor: Computation of Bayes factors for common designs. Retrieved from https://CRAN.R-project.org/package=BayesFactor

Osth, A. F., Bora, B., Dennis, S., \& Heathcote, A. (2017). Diffusion vs. linear ballistic accumulation: Different models, different conclusions about the slope of the zROC in recognition memory. Journal of Memory and Language, 96, 36-61. https://doi.org/10.1016/j.jml.2017.04.003

R Core Team. (2022). R: A language and environment for statistical computing. Vienna, Austria: R Foundation for Statistical Computing. Retrieved from https://www.R-project.org/ 
Rabe, M. M., Lindsay, D. S., \& Kliegl, R. (2021, March 31). ROC asymmetry is not diagnostic of unequal residual variance in Gaussian signal detection theory. https://doi.org/10.31234/osf.io/erzvp

Ratcliff, R., McKoon, G., \& Tindall, M. (1994). Empirical generality of data from recognition memory receiver-operating characteristic functions and implications for the global memory models. Journal of Experimental Psychology: Learning, Memory, and Cognition, 20(4), 763-785. https://doi.org/10.1037/0278-7393.20.4.763

Ratcliff, R., Sheu, C.-F., \& Gronlund, S. D. (1992). Testing global memory models using ROC curves. Psychological Review, 99(3), 518-535. https://doi.org/10.1037/0033295X.99.3.518

Ratcliff, R., \& Starns, J. J. (2009). Modeling confidence and response time in recognition memory. Psychological review, 116(1), 59-83. https://doi.org/10.1037/a0014086

Ratcliff, R., \& Starns, J. J. (2013). Modeling confidence judgments, response times, and multiple choices in decision making: recognition memory and motion discrimination. Psychological review, 120(3), 697-719. https://doi.org/10.1037/a0033152

Rotello, C. (2017). Signal detection theories of recognition memory. In J. T. Wixted (Ed.), Learning and Memory: A Comprehensive Reference (2nd ed., Vol. 4., pp. 201-225). Elsevier. https://doi.org/10.1016/B978-0-12-809324-5.21044-4

Spanton, R. W., \& Berry, C. J. (2020). The unequal variance signal detection model of recognition memory: Investigating the encoding variability hypothesis. Quarterly Journal of Experimental Psychology, 73(8), 1242-1260. https://doi.org/10.1177/1747021820906117 
Starns, J. J., Ratcliff, R., \& McKoon, G. (2012). Evaluating the unequal-variance and dualprocess explanations of zROC slopes with response time data and the diffusion model. Cognitive Psychology, 64(1-2), 1-34. https://doi.org/10.1016/j.cogpsych.2011.10.002

Starns, J. J., Rotello, C. M., \& Ratcliff, R. (2012). Mixing strong and weak targets provides no evidence against the unequal-variance explanation of zROC slope: A comment on Koen and Yonelinas (2010). Journal of Experimental Psychology: Learning, Memory, and Cognition, 38(3), 793-801. https://doi.org/10.1037/a0027040

Van Heuven, W. J., Mandera, P., Keuleers, E., \& Brysbaert, M. (2014). SUBTLEX-UK: A new and improved word frequency database for British English. The Quarterly Journal of Experimental Psychology, 67(6), 1176-1190.

Yonelinas, A. P., \& Parks, C. M. (2007). Receiver operating characteristics (ROCs) in recognition memory: a review. Psychological bulletin, 133(5), 800-832. https://doi.org/10.1080/17470218.2013.850521

Wickelgren, W. A. (1968). Unidimensional strength theory and component analysis of noise in absolute and comparative judgments. Journal of Mathematical Psychology, 5(1), 102122. https://doi.org/10.1016/0022-2496(68)90059-X

Wickham, H., Averick, M., Bryan, J., Chang, W., McGowan, L. D., François, R., ... Yutani, H. (2019). Welcome to the tidyverse. Journal of Open Source Software, 4(43), 1686. https://doi.org/10.21105/joss.01686

Wixted, J. T. (2007). Dual-process theory and signal-detection theory of recognition memory. Psychological Review, 114(1), 152. https://doi.org/10.1037/0033-295X.114.1.152

Yonelinas, A. P. (1994). Receiver-operating characteristics in recognition memory: evidence for a dual-process model. Journal of Experimental Psychology: Learning, Memory, and Cognition, 20(6), 1341-1351. https://doi.org/10.1037/0278-7393.20.6.1341 
Yonelinas, A. P., \& Parks, C. M. (2007). Receiver operating characteristics (ROCs) in recognition memory: A review. Psychological Bulletin, 133(5), 800-832.

https://doi.org/10.1037/0033-2909.133.5.800 


\section{Appendix A: Parameter Recovery Simulation for Experiment 3}

A total of 11 free parameters are required to simultaneously model the four conditions in Experiment 3 with our extended UVSD model specification. This is four more than was required to model each test phase in Experiments 1 and 2, and it is therefore important to determine whether reliable estimates of the free parameters can be recovered. To do this, 75 datasets with the same number of trials as data from Experiment 3 (240 total) were simulated from, and the UVSD model was fit to these datasets to recover the true generative parameters. The datasets were generated using the UVSD model's parameter specification; for each free parameter, values were drawn from a uniform distribution to evenly cover a plausible parameter space. The bounds on these uniform distributions and the mean values of the true and estimated parameters can be found in Table A1. The bounds upon the criteria distributions were defined in a way that ensured each successive criterion was greater than the last.

Bayesian $t$-tests found a strong absence of a difference between estimated and true values for all the model parameters $(\mathrm{BFs} \leq 0.33)$ except for $\mu_{\mathrm{oh}}, \mu_{\mathrm{ol}}, \sigma_{\mathrm{ol}}$, where inconclusive evidence was found, although these Bayes Factors were still relatively low. This indicates that the model can successfully estimate true parameter values in simulated data with the same number of trials and participants as in Experiment 3. It is of note that some outlying estimates were produced in this simulation, which reflect in the mean and standard deviation of certain parameter estimates. Although these outliers make the means less representative of the model's general predictions about data (and are therefore excluded in our experiments), the model can still produce a generally accurate account of the true parameters in simulated data with these outliers included. 
Table A1.

Generative lower and upper bounds $\mathrm{a}$ and $\mathrm{b}$ on the uniform distributions used to simulate true parameters, the means of the true and estimated parameters given by the extended UVSD model (SDs in parentheses), and the Bayes Factor of the comparisons between true and estimated parameter values.

\begin{tabular}{llllllll} 
Parameter & $a$ & $b$ & \multicolumn{2}{l}{ True Mean } & \multicolumn{2}{l}{ Mean Estimate } & BF \\
\hline$\mu_{\mathrm{nh}}$ & -1 & 1 & -0.08 & $(0.56)$ & -0.02 & $(0.73)$ & 0.20 \\
$\sigma_{\mathrm{nh}}$ & 0.5 & 4 & 2.30 & $(0.98)$ & 2.50 & $(1.43)$ & 0.27 \\
$\mu_{\mathrm{oh}}$ & 0 & 4 & 2.14 & $(1.08)$ & 3.12 & $(6.59)$ & 0.37 \\
$\sigma_{\mathrm{oh}}$ & 0.5 & 4 & 2.45 & $(1.02)$ & 2.56 & $(1.45)$ & 0.20 \\
$\mu_{\mathrm{ol}}$ & 0 & 4 & 1.94 & $(1.19)$ & 2.93 & $(5.71)$ & 0.47 \\
$\sigma_{\mathrm{ol}}$ & 0.5 & 4 & 2.33 & $(1.03)$ & 2.66 & $(1.90)$ & 0.40 \\
$C_{1}$ & -1 & 0.2 & -0.42 & $(0.35)$ & -0.45 & $(0.43)$ & 0.20 \\
$C_{2}$ & $C_{1}+0.01$ & $C_{1}+1$ & 0.09 & $(0.42)$ & 0.10 & $(0.48)$ & 0.18 \\
$C_{3}$ & $C_{2}+0.01$ & $C_{2}+1$ & 0.61 & $(0.53)$ & 0.63 & $(0.58)$ & 0.18 \\
$C_{4}$ & $C_{3}+0.01$ & $C_{3}+1$ & 1.18 & $(0.58)$ & 1.23 & $(0.67)$ & 0.20 \\
$C_{5}$ & $C_{4}+0.01$ & $C_{4}+1$ & 1.68 & $(0.64)$ & 1.75 & $(0.75)$ & 0.21 \\
\hline
\end{tabular}

\section{Maximum Likelihood Estimation Fit Procedure}

As in the rest of our experiments, the UVSD model was fit to data using maximum likelihood estimation. To fit the model to a given participant's data in Experiment 3, initial starting estimates of each of the model's free parameters were derived from the data. The parameters were derived using the approximations detailed in the supplemental materials of Spanton and Berry (2020), with $d^{\prime}$ being used to approximate both old item distribution means, and $1 / z$-ROC slope used as an approximation of the standard deviation of both old item distributions. These estimated starting parameters were used to sample ten sets of starting parameters from normal distributions. Another twenty sets of starting parameters were sampled from normal and uniform distributions with means, standard deviations and bounds that were not estimated from participant data. This was intended to provide a broad range of plausible starting 
parameters to avoid local minima in the model fitting procedure. According to the method described by Dunn (2010), the non-negative starting values were then log transformed, and all were input to the likelihood function which was optimized using the Nelder-Mead algorithm (as implemented in the optim function in R), giving 30 model fits per participant. The best of these model fits - that with the greatest log-likelihood - was chosen for each participant and used to give their parameter estimates.

The log-likelihood function for the UVSD model in Experiment 3 is

$$
\sum_{j=1}^{4} \sum_{i=1}^{6} N_{i, j} \times \log \left[\Phi\left(I_{i+1}, \mu_{j}, \sigma_{j}\right)-\Phi\left(I_{i}, \mu_{j}, \sigma_{j}\right)\right]
$$

where $i$ is the criterion index, $j$ represents each condition (old-high, old-low, new-high, new-low), $\Phi$ is the normal cumulative distribution function, and $I=\left\{-\infty, C_{1}, C_{2}, \ldots, C_{\mathrm{I}}, \infty\right\}$ is a vector of criteria with upper and lower bounds of positive and negative infinity. 